\title{
LabVIEW Interactive Simulations for Electromagnetic Compatibility
}

http://dx.doi.org/10.3991/ijoe.v8i2.1935

\author{
E. LUNCA, S. URSACHE and A. SALCEANU \\ Technical University of Iasi, Romania
}

\begin{abstract}
At university level, virtual instrumentation techniques are extensively used for both education and research in various fields of electrical engineering (EE). In this paper, we illustrate the possibility of using a virtual instrumentation approach focused on LabVIEW to create interactive simulations for Electromagnetic Compatibility (EMC). Firstly, the main reasons for adopting the LabVIEW graphical programming to develop such tools are given. Then, a number of specific modules are presented.
\end{abstract}

Index Terms—simulations, virtual instruments, LabVIEW, electromagnetic compatibility.

\section{INTRODUCTION}

During the past two decades, LabVIEW - a fullyfeatured graphical programming language from National Instruments - has been used by millions of engineers and scientists to develop sophisticated measurement, test and control systems. It eliminates a lot of the syntactical details associated with text-based languages, while delivering extensive data acquisition, analysis and presentation in a single environment.

In academia, LabVIEW has capabilities to easy integrate with the leading tools used both in research and education [1-5]. Thanks to its advanced simulation capability and intuitive interfaces, LabVIEW allows conducting realistic EE experiments even if there is no access to real equipment [6-9]. For such a reason, we decided to use LabVIEW for building simulation models intended to illustrate EMC concepts. Certainly, this is not a usual approach, but, with minimum cost and programming efforts, an interactive experience based on "virtual instruments" (VIs) may greatly reinforce the EMC theory.

Among others, the benefits of using LabVIEW to develop computer simulations for EMC include:

- possibility to build accurate mathematical models describing various phenomena and systems;

- attractive graphical user interfaces (GUIs), which allow users to fully interact with the simulated phenomena;

- reduced development time and complexity due to configurable Express VIs and ready-to-use example VIs;

- easy update with new requirements and developments;

- scientific visualization capability including specialized plots and graphs (Smith, Bode, polar, 3D curve graphs, etc.);
- existence of dedicated modules and toolkits, such as Control Design and Simulation Module, Simulation Interface Toolkit (connects LabVIEW user interfaces with simulation models running in Simulink), Spectral Measurements Toolkit and Modulation Toolkit, which can extend the basic functionality of LabVIEW to develop virtual instruments concerning EMC and EMC-related topics;

- possibility to create executable applications with the LabVIEW Application Builder and to run them on any computer using the LabVIEW Run-Time Engine, a small standalone program that can be downloaded from the NI website.

It is also worthwhile to mention that, since the LabVIEW built-in Web Server allows controlling any VI front panel directly from a web browser, an on-line experience for EMC can be achieved in just a few simple steps. As in the case of Java applets, running a LabVIEW program over the Internet will require client computers to have installed only LabVIEW Run-Time Engine.

\section{READY-TO-USE LABVIEW EXAMPLES FOR EMC}

LabVIEW integrates hundreds of example VIs that can be used for performing different types of EE experiments. FFT and Frequency Analysis, Distortion Measurements, Time-Domain Analysis and Filtering are popular categories of built-in VIs that may serve as demonstrative models for illustrating EMC-related concepts, such as spectral content of signals, spectral measurements, THD and SINAD measurements, noise waveforms and PS density, etc. Note that most of these programs are simulations.

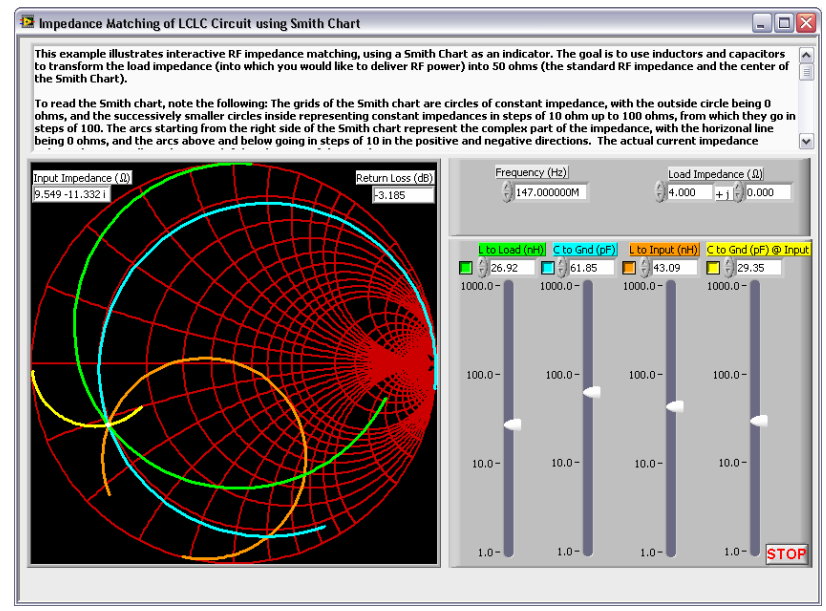

Figure 1. Simulation LabVIEW program for interactive RF impedance matching [10] 
However, using LabVIEW to explore specific EMC concepts requires developing our own code. Even if there are a huge number of examples available on the NI website, only a few of them are intended to explore EMC concepts. Figure 1 presents the front panel of such a program, which utilizes a Smith chart to match impedances of a common LCLC circuit. The component parameters are set interactively and the resulting impedance circles are shown instantaneously [10].

\section{DEVELOPING LABVIEW SimULATIONS FOR EMC}

In order to reinforce the EMC theory, a series of LabVIEW interactive simulations have been created by the authors to illustrate EMC and EMC-related topics, including: simple spectrum analyzer based on Zoom FFT, analog modulations (AM, FM), PCB impedance control, lossless transmission line behavior, polarization of electromagnetic waves, field-strength and power estimation (in far field conditions), radiation properties of arbitrary length dipole antenna, radiation properties of antenna arrays, radiation properties of aperture antennas (open ended rectangular waveguide), electromagnetic radiation from uniform apertures (rectangular and circular), electromagnetic radiation from straight interconnect cables, etc. Some of these achievements will be presented in the following, whereas a few others have been reported in a prior work [11].

\section{A. Using Express VIs to develop simple models. Case study: Amplitude Modulation}

As stated earlier in this paper, LabVIEW integrates configuration-based Express VIs for measurement analysis and signal processing. By requiring little or no programming, they may greatly reduce the development time and complexity associated with adding analysis and signal processing algorithms into applications. For instance, a virtual instrument intended to explore the amplitude modulation - important principle helping students to understand the way in which the electromagnetic energy is transmitted - can be developed in just a few steps, by following the simple approach given below.

Let's consider the analytical expression of an amplitude modulated signal:

$$
y(t)=A_{c}\left(1+m \cos \omega_{m} t\right) \cos \omega_{c} t,
$$

where $A_{c}$ and $\omega_{c}$ are parameters of the carrier signal, $\omega_{m}$ is the frequency of the modulating signal and $m$ is the modulation index.

Starting with this equation, $y(t)$ can be trigonometrically manipulated into the equivalent form:

$$
\begin{aligned}
& y(t)=A_{c} \cos \omega_{c} t+\frac{m A_{c}}{2} \cos \left(\omega_{c}+\omega_{m}\right) t+ \\
& +\frac{m A_{c}}{2} \cos \left(\omega_{c}-\omega_{m}\right) t
\end{aligned},
$$

which indicates that the amplitude modulated signal has three components. First of them has the same frequency as the unmodulated carrier, the second component has a frequency equal to the sum of the carrier frequency and the modulation frequency, $\omega_{c}+\omega_{m}$, while the third component has a frequency equal to the difference between the carrier frequency and the modulation frequency, $\omega_{c}-\omega_{m}$.

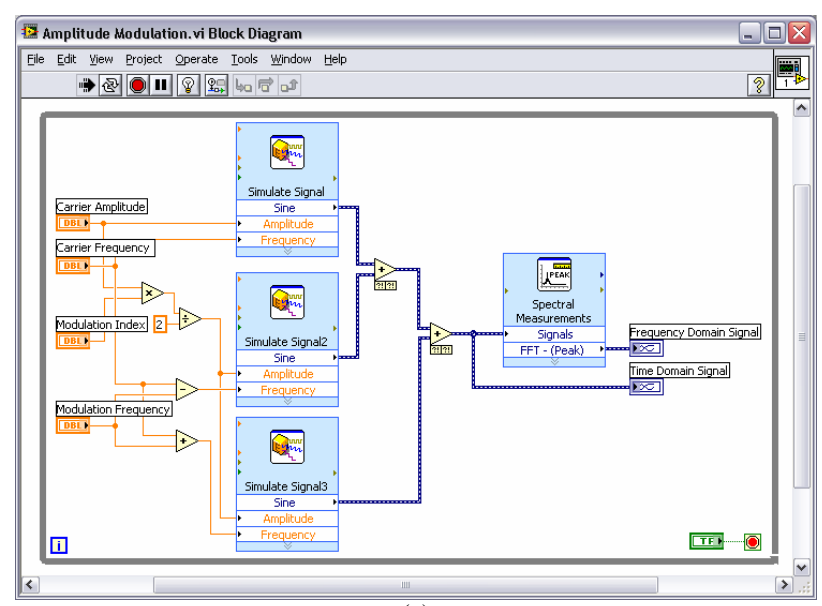

(a)

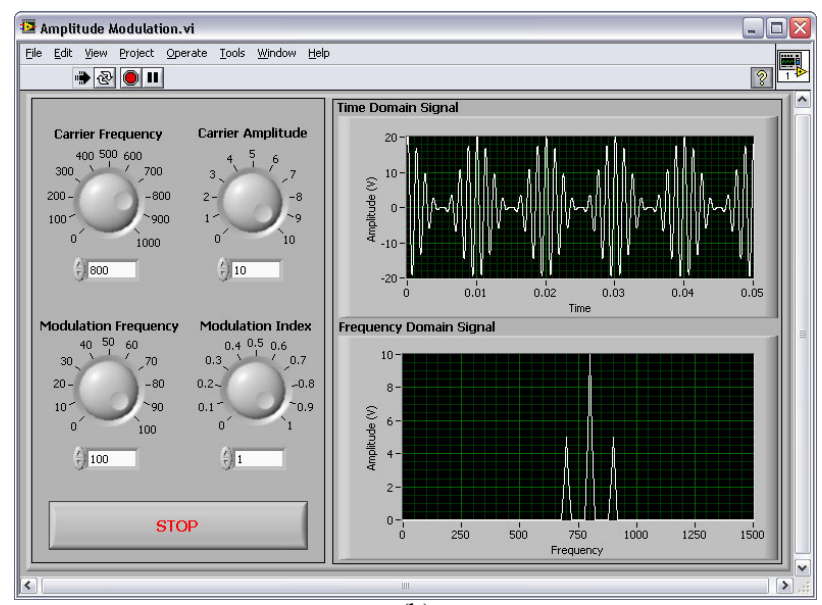

(b)

Figure 2. Virtual instrument for simulating the amplitude modulation: (a) block diagram; (b) front panel

As illustrated in Figure 2.a, the described mathematical model can be simply implemented in LabVIEW by using three "Simulate Signal" Express VIs, configured with adequate information of amplitude, frequency and phase, and having the outputs added together, according to the equation (2). Furthermore, a "Spectral Measurements" Express VI computes the spectrum of the synthesized AM signal and displays the results in terms of peak value.

The graphical user interface in Figure 2.b allows exploring the AM modulation by adjusting all parameters of the mathematical model through either knob or numeric controls. For example, the actual measurement screen corresponds to a modulation index of 1 , which is the maximum value allowed in the AM transmission systems; varying $m$ beyond this value will enable users to observe the overmodulation condition that, in telecommunications, should be usually avoided because of its attendant distortion and interference problems.

\section{B. Exploring the lossless transmission line behavior}

An understanding of the transmission line theory is essential not only in the RF communications and digital circuit design, but also in the Electromagnetic Compatibility. It provides a link between circuit analysis and electromagnetics.

Figure 3 presents a LabVIEW simulation tool, which is designed to analyze the behavior of a lossless transmission line connected between a generator and a load. 
PAPER

LABVIEW INTERACTIVE SIMULATIONS FOR ELECTROMAGNETIC COMPATIBILITY

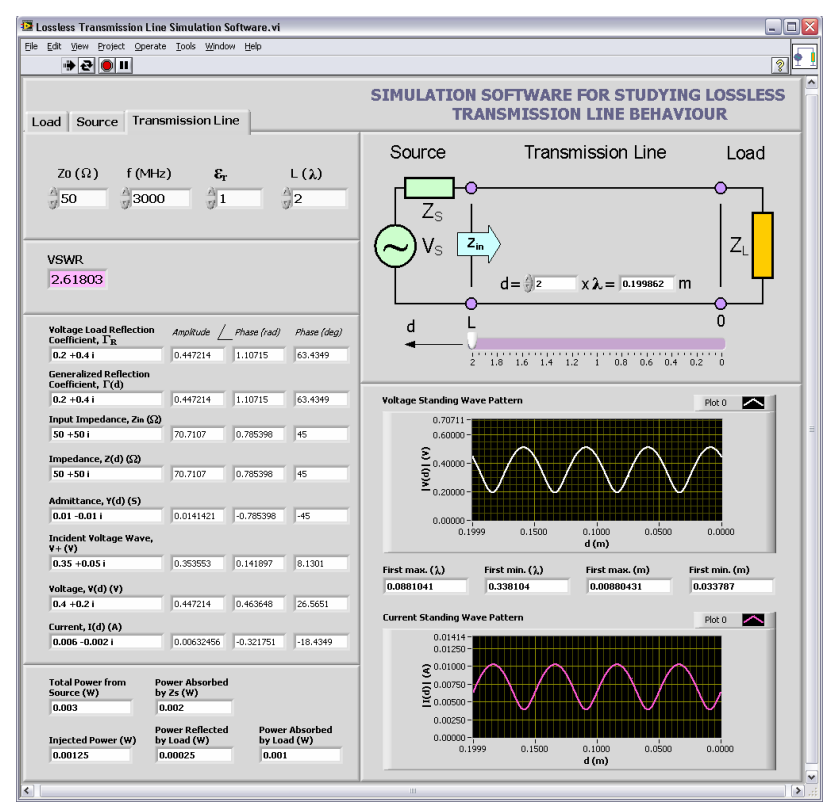

Figure 3. Simulation tool for studying the behavior of the lossless transmission line

Basically, it computes a series of parameters associated with the sinusoidal excitation of the transmission line (TL) and plots the voltage and current standing wave patterns along the TL. The line is assumed to have the arrangement and coordinate system presented in the upper right side of the graphical user interface, with the cursor position varying between 0 (at the load) and $\mathrm{L}$ (at the source).

The input data are introduced through the three tabs located in the upper left side of the GUI, as following:

- Load - allows specifying the complex impedance of the load $Z_{L}$;

- Source - allows specifying the complex source voltage $U_{S}$ and complex source impedance $Z_{S}$;

- Transmission line - allows specifying the characteristic impedance of the line $Z_{0}$, frequency $f$, relative dielectric constant of the material between the conductors $\varepsilon_{r}$ and line length $L$ (as a function of wavelength $\lambda$ ).

Based on these data and - where appropriate - on the actual position of the cursor $(d)$, the program interactively displays the following TL parameters:

- voltage standing wave ratio, VSWR;

- voltage reflection coefficient at the load, $\Gamma_{R}$;

- generalized reflection coefficient, $\Gamma(d)$ (for the cursor position $d=0$, this parameter is equal to $\Gamma_{R}$ );

- input impedance to the line, $Z_{\text {in }}$;

- impedance along the line, $Z(d)$ (for the cursor position $d=L$, this parameter is equal to $\left.Z_{i n}\right)$;

- admittance along the line, $Y(d)$;

- amplitude of the incident voltage wave, $V+$;

- voltage along the line, $V(d)$;

- current along the line, $I(d)$;

- time-average power, including total power from source, power absorbed by the source impedance; injected power, power reflected by load and power absorbed by load.
As it can easily be seen in the "parameter display area", the phasorial quantities are reported in both rectangular and polar form. Note that, in the case of the polar representation (which implies magnitude and phase information), the phase angle is expressed in both radians and degrees.

Finally, the voltage and current standing wave patterns are also displayed on the two graphs located under the TL set-up. They provide the top envelopes that bound the time-oscillations of the voltage and current along the line or, in other words, the maximum values that the voltage and current can establish at each location of the TL for a given load and generator, because of the interference between the incident and reflected wave. For voltage, the distance of first maximum and distance of first minimum (from the load) are indicated under the correspondent graph, both as a function of $\lambda$ and in units of $\mathrm{m}$.

Generally, this simulation tool can be used to quickly solve various problems related to the lossless TL or to illustrate fundamental TL concepts, such as impedance matching, standing (stationary) waves, power flow on lossless line, etc. Comparisons to other sources have showed that it produces very accurate results - the output data and standing wave patterns associated with various transmission line configurations are identical to those produced by some JAVA applets or those given in textbooks [12, 13].

\section{C. $R F$ radiation from uniform apertures}

A simulation program for investigating the electromagnetic radiation from uniform rectangular and circular apertures is shown in Figure 4. The polar angle conventions assumed for this VI are depicted in the upper left side of the GUI, with the origin being placed in the middle of the aperture.

In the case of the rectangular aperture, the program plots the E-field strength pattern along the two principal planes, $x z\left(\Phi=0^{\circ}\right)$ and $y z\left(\Phi=90^{\circ}\right)$, as a function of the geometrical dimensions of the aperture, $a(\lambda)$ and $b(\lambda)$. Dictated by the sinc function properties, these representations will have narrower main beams, as well as narrower side lobes, as the aperture size is increased. The particular pattern in Figure 4 (front window) corresponds to $a=8 \lambda$ and $b=4 \lambda$, and features an obvious $x-y$ dissimilarity.

In the case of the circular aperture, the program plots the E-field strength pattern along the two principal planes as a function of the aperture radius, $a(\lambda)$. Since this pattern is described by the Bessel function of the first kind of order 1, it is simply computed by using the Bessel Function Jv VI included in the Full Development System. As expected from theory, for large circular apertures, the $x y$ and $x z$-plane patterns are very similar. In other words, the correspondent 3D representation, which may be obtained by mentally putting these graphs together, features a fairly symmetrical beam. The particular pattern in Figure 4 (back window) corresponds to $a=3 \lambda$ and consists of a narrow main lobe, directed towards the forward direction $\theta=0^{\circ}$, and a few side lobes.

In addition to this program, a version that computes the radiation patterns of the two apertures in polar coordinates has also been developed. For instance, Figure 5 presents the principal patterns for the rectangular aperture of size $a$ $=3 \lambda$ and $b=2 \lambda$. And this time, the results produced with both programs are consistent with those obtained from other reference sources [14, 15]. 
PAPER

LABVIEW INTERACTIVE SIMULATIONS FOR ELECTROMAGNETIC COMPATIBILITY

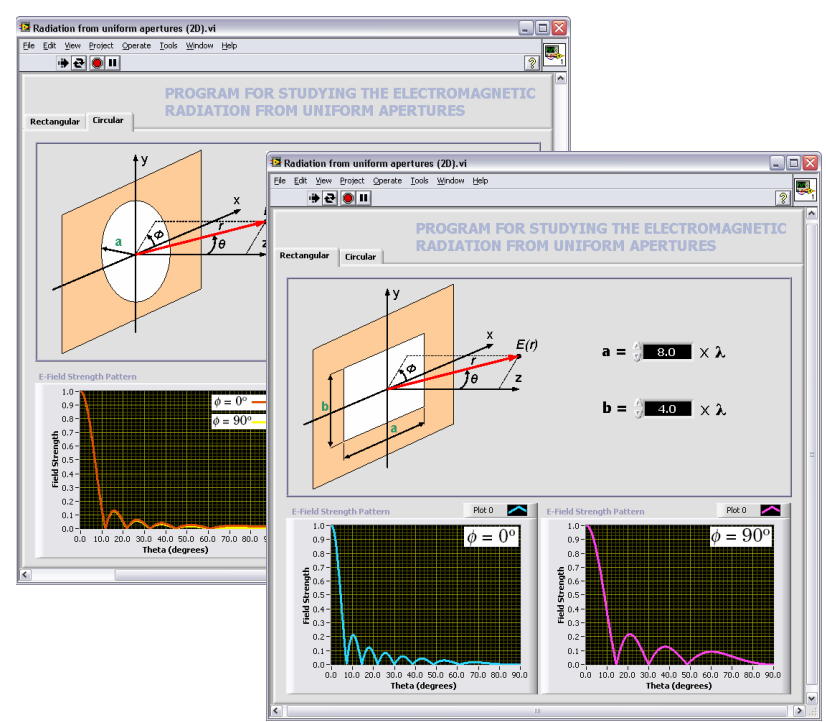

Figure 4. Simulation program for studying the RF radiation from uniform apertures

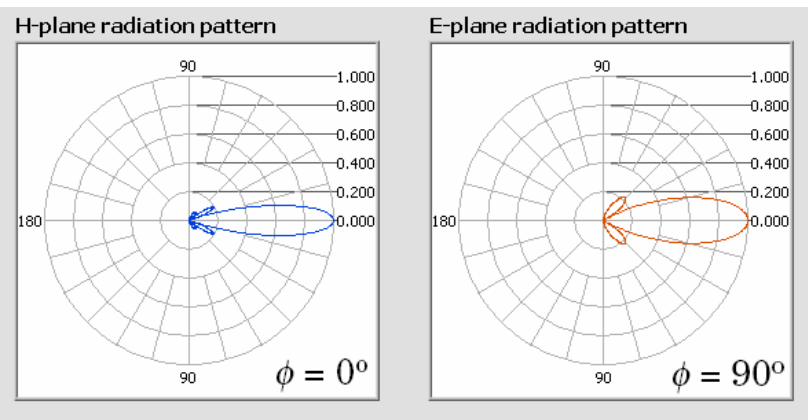

Figure 5. The radiation pattern of the rectangular aperture in polar coordinates $(a=3 \lambda$ and $b=2 \lambda)$

\section{CONCLUSIONS AND FUTURE WORK}

This paper has shown that using LabVIEW is feasible to develop inexpensive, feature-rich simulation tools for exploring various EMC and EMC-related concepts. Because of their intuitive and interactive nature, the LabVIEW VIs may provide students or anyone interested in the EMC area with a truly "realistic virtual experience" prior, or in addition, to any hands-on training.

Our long-term interest is to enhance and extend this work in order to provide a more complex and effective EMC learning environment. Feedback from other teachers and researchers is welcome as it can lead to further improvements and developments of these tools. Note, only a part of them have been presented here.

\section{ACKNOWLEDGMENT}

This paper was supported by the project PERFORMERA "Postdoctoral Performance for Integration in the European Research Area” (ID-57649), financed by the European Social Fund and the Romanian Government.

\section{REFERENCES}

[1] Y. Ugurlu, "Measuring the Impact of Virtual Instrumentation for Teaching and Research", 2011 IEEE Global Engineering Education Conference (EDUCON) - "Learning Environments and Ecosystems in Engineering Education”, 2011, pp. 152-158.

[2] Tutorial "Advantages of Using LabVIEW in Academic Research", available online at the address http://zone.ni.com/devzone/ cda/tut/p/id/8534.

[3] A. Odon, Z. Krawiecki, "Using LabVIEW for Computer Modelling of Conversion Methods in Analog to Digital Converters”, $12^{\text {th }}$ IMEKO TC1 \& TC7 Joint Symposium on Man Science \& Measurement, 2008, pp. 395-400.

[4] A.N. D’Andrea, F. Giannetti, G. Manara, M. Michelini, P. Nepa, "Educational Set-Up for Microwave Laboratory Activities Using Virtual Instruments and Real Data", in Microwave Review, Vol. 13, No. 2, 2007, pp. 12-17.

[5] L. Sevgi, C. Uluisik, "A Labview-Based Virtual Instrument for Engineering Education: A Numerical Fourier Transform Tool", in Turkish Journal of Electrical Engineering \& Computer Sciences, Vol. 14, No. 1, 2006, pp. 129-152.

[6] N.K. Swain, J.A. Anderson, "Study of Electrical Power Systems Using LabVIEW Virtual Instruments (VI) Modules”, IAJC-IJME International Conference, 2008.

[7] M. Usama Sardar, "Synchronous Generator Simulation Using LabVIEW", Proceedings of World Academy of Science, Engineering \& Technology (WASET), Vol. 29, 2008, pp. 392-400.

[8] C. Fosalau, L. Nita, M. Antoniu, "Virtual instrument for digital signal processing learning", $3^{\text {rd }}$ International Conference on Electrical and Power Engineering, 2006.

[9] E. Brenner, "Virtual Instruments for the Circuit Analysis with Interactive Parameter Modification", in International Journal of Online Engineering (iJOE), Vol. 2, No. 3, 2006.

[10] Example program "Impedance Matching using Smith Charts", available online at the address http://zone.ni.com/devzone/ cda/epd/p/id/3604.

[11] E. Lunca, A. Salceanu, "Virtual Instrumentation Approach for Teaching EMC Concepts", in Electronics and Electrical Engineering, No. 1(117), 2012, pp. 75-80.

[12] Interactive Java applets available online at the address http://www.amanogawa.com/archive/transmissionA.html.

[13] F.T. Ulaby, Fundamentals of Applied Electromagnetics $\left(5^{\text {th }}\right.$ Edition), Prentice Hall, 2006.

[14] Interactive Java applets available online at the address http://www.analyzemath.com/antenna_tutorials/aperture_antennas. html.

[15] N.K. Nikolova, "Modern Antennas in Wireless Telecommunications", Course available online at the address http://www.ece.mcmaster.ca/faculty/nikolova/antennas.htm.

\section{AUTHORS}

Eduard Lunca is a lecturer with the Electrical Measurements Department, Faculty of Electrical Engineering, Iasi, Romania (elunca@ee.tuiasi.ro).

Silviu Ursache is a teaching assistant with the Electrical Measurements Department, Faculty of Electrical Engineering, Iasi, Romania (silviu_ursache@ee.tuiasi.ro).

Alexandru Salceanu is a full professor with the Electrical Measurements Department, Faculty of Electrical Engineering, Iasi, Romania. At the moment, he is the dean of this faculty (asalcean@ee.tuiasi.ro).

Received 16 January 2012. Published as submitted by the authors 27 April 2012. 